\title{
RESEARCH
}

Open Access

\section{Enrichment differentiation of human induced pluripotent stem cells into sinoatrial node-like cells by combined modulation of BMP, FGF, and RA signaling pathways}

Feng Liu ${ }^{1+}$, Yibing Fang ${ }^{1+}$, Xiaojie Hou', Ying Yan², Haiying Xiao ${ }^{3}$, Dongchuan Zuo ${ }^{3}$, Jing Wen ${ }^{3}$, Linli Wang ${ }^{4}$, Zhichao Zhou ${ }^{5}$, Xitong Dang ${ }^{3}$, Rui Zhou ${ }^{3^{*}}$ and Bin Liao ${ }^{1^{*}}$

\begin{abstract}
Background: Biological pacemakers derived from pluripotent stem cell (PSC) have been considered as a potential therapeutic surrogate for sick sinus syndrome. So it is essential to develop highly efficient strategies for enrichment of sinoatrial node-like cells (SANLCs) as seed cells for biological pacemakers. It has been reported that BMP, FGF, and RA signaling pathways are involved in specification of different cardiomyocyte subtypes, pacemaker, ventricular, and atrial cells. We aimed to investigate whether combined modulation of BMP, FGF, and RA signaling pathways could enrich the differentiation of SANLC from human pluripotent stem cell (hiPSC).

Methods: During the differentiation process from human induced pluripotent stem cell to cardiomyocyte through small molecule-based temporal modulation of the Wnt signaling pathway, signaling of BMP, FGF, and RA was manipulated at cardiac mesoderm stage. QRT-PCR, immunofluorescence, flow cytometry, and whole cell patch clamp were used to identify the SANLC.

(Continued on next page)
\end{abstract}

\footnotetext{
*Correspondence: zhouhuaxizhu@swmu.edu.cn; 1875153677@qq.com

${ }^{\dagger}$ Feng Liu and Yibing Fang contributed equally to this work.

${ }^{3}$ The Key Laboratory of Medical Electrophysiology of Ministry of Education

and Medical Electrophysiological Key Laboratory of Sichuan Province,

Collaborative Innovation Center for Prevention and Treatment of

Cardiovascular Disease of Sichuan Province, Institute of Cardiovascular

Research, Southwest Medical University, 3-319 Zhongshan Road, Luzhou

646000, Sichuan, China

'Department of Cardiac Macrovascular Surgery, Affiliated Hospital of

Southwest Medical University, 3-319 Zhongshan Road, Luzhou 646000,

Sichuan, China

Full list of author information is available at the end of the article
}

C C The Author(s). 2020 Open Access This article is licensed under a Creative Commons Attribution 4.0 International License, which permits use, sharing, adaptation, distribution and reproduction in any medium or format, as long as you give appropriate credit to the original author(s) and the source, provide a link to the Creative Commons licence, and indicate if changes were made. The images or other third party material in this article are included in the article's Creative Commons licence, unless indicated otherwise in a credit line to the material. If material is not included in the article's Creative Commons licence and your intended use is not permitted by statutory regulation or exceeds the permitted use, you will need to obtain permission directly from the copyright holder. To view a copy of this licence, visit http://creativecommons.org/licenses/by/4.0/ The Creative Commons Public Domain Dedication waiver (http://creativecommons.org/publicdomain/zero/1.0/) applies to the data made available in this article, unless otherwise stated in a credit line to the data. 
(Continued from previous page)

Results: qRT-PCR results showed that manipulating each one of bone morphogenetic protein (BMP), fibroblast growth factor (FGF), and retinoid acid (RA) signaling was effective for the upregulation of SANLC markers. Moreover, combined modulation of these three pathways displayed the best efficiency for the expression of SANLC markers, which was further confirmed at protein level using immunofluorescence and flow cytometry. Finally, the electrophysiological characteristics of upregulated SANLC were verified by patch clamp method.

Conclusion: An efficient transgene-independent differentiation protocol for generating SANLC from hiPSC was developed, in which combined modulating BMP, FGF, and RA signaling at cardiac mesoderm stage generates SANL $C$ at high efficiency. This may serve as a potential approach for biological pacemaker construction.

Keywords: Human induced pluripotent stem cell (hiPSC), Sinus atrial node-like cell (SANLC), Bone morphogenetic protein (BMP) signaling, Fibroblast growth factor (FGF) signaling, Retinoic acid (RA) signaling

\section{Introduction}

"Sick sinus syndrome" (SSS) is a group of heart arrhythmia caused by perturbed function of the sinus node that is composed of cardiac pacemaker cells. They include pathological and/or symptomatic sinus bradycardia, sinoatrial (SA) block, and tachycardia-bradycardia syndrome. Although implantation of electronic pacemaker has been one of the most effective treatments at present, it is associated with significant risks of infection, hemorrhage, and lead dislodging. Moreover, limited battery life and lack of autonomic neurohumoral responses further limit its usage $[1,2]$. Therefore, the "biological pacemaker" derived from human pluripotent stem cells (hPSCs) may provide a promising alternative treatment.

Cardiomyocyte differentiation from hPSC is recognized as a powerful model to simulate the human embryonic heart development in vivo and is a promising source of cardiomyocytes for regenerative medicine. The embryonic heart development experiences several spatial-temporal stages from primitive streak to cardiac crescent and then to primitive heart tube, and the latter forms distinct anterior and posterior poles containing different mesodermal progenitors that give rise to different cardiomyocyte subtypes including ventricular, atrial, and sinoatrial node (SAN)-like cardiomyocytes (SANL Cs) [3-5]. To date, most protocols were designed to generate heterogenous ventricular cardiomyocytes with a very small percentage of atrial and SANLC [6, 7]. Analyses of early developmental stages revealed that ventricular, atrial, and SAN-like cardiomyocytes were derived from different mesoderm cell populations that could be distinguished based on their expression of CD235a [8, 9], RALDH2 [8], and TBX18 [10-12], respectively. In addition, it was found that different signaling pathways were involved in different cardiomyocyte subtype specifications. For example, retinoic acid (RA) signaling at the mesoderm stage is required for atrial specification [8], while ventricular specification is highly dependent on the fibroblast growth factor (FGF) signaling [13-16]. A recent study showed that bone morphogenetic protein (BMP) signaling plays an important role in the specification of mesoderm progenitors into SANLC [17, 18]. These early discoveries suggest that hiPSC-induced cardiomyocytes can be directed to differentiate into SAN-like cardiomyocytes at cardiac mesoderm stage by activation of the BMP signaling pathways leading to SAN-like, and by simultaneous inhibition of signaling pathways leading to ventricular and atrial cardiomyocytes.

Consequently, we aimed to figure out a new working model of enriched differentiation of SANLC from hiPSC. We have established a developmental biology-based approach to enrich SANLC from hiPSC. We found in the present study that activation of BMP and simultaneous inhibition of RA and FGF signaling pathways during cardiac mesoderm stage of hiPSC differentiation can significantly enrich SAN-like cardiomyocytes. These findings will facilitate the study of human SAN development and may provide a rich source of cells for the development of biological pacemaker.

\section{Materials and methods Materials}

The information of purchased reagents and cell line used in the study is as follows: LHpb-YaabC3 hiPSC (HNFP30-P11, OSINGLAY BIO, China), BJ human foreskin fibroblast cell line (CRL2522, ATCC, USA), HN4 human embryonic stem cell (hESC) line (HES-P20-P9, OSINGLAY BIO, China), Cell culture media RPMI/1640 (11875093, Thermo Fisher Scientific, USA), DMEM/F12 media (11320082, Thermo Fisher Scientific, USA), medium for hiPSC BioCISO (BC-PM0001, OSINGLAY BIO, China), GSK3 inhibitor CHIR99021 (S1263, Sigma, USA), FGF inhibitor PD (3044, Tocris Bioscience, UK), Wnt inhibitor IWP2 (3533, Tocris Bioscience, UK), ROCK inhibitor Y27632 (1254, Tocris Bioscience, UK), BMP activator BMP4 (120-05ET, PEPROTECH, USA), RA inhibitor BMS (SML1149, Sigma, USA), B-27 supplement with (17504-044, Thermo Fisher Scientific, USA) or without insulin, Matrigel (354277, Corning, UK), 
TRIzol Reagent (15596026, Thermo Fisher Scientific, USA), and Real-time PCR reagents (208056, Qiagen, Germany). All primers/oligos were synthesized by Shenggong Biotech, China, and listed in Table 1. All other reagents, unless specified otherwise, were products of Sigma.

\section{Cardiomyocyte differentiation from hiPSC}

Cardiomyocyte differentiation was performed in a growth factor and serum-free system by temporal modulation of the canonical Wnt signaling pathway with GSK3 inhibitor (Gi) and Wnt inhibitor (Wi), known as the GiWi protocol [19]. Briefly, 80-90\% confluent hiPSC was harvested using $0.5 \mathrm{mM}$ EDTA and resuspended with hiPSC-maintaining medium at $0.5 \times 10^{5}$ cells per milliliter. Two milliliters of the cell suspension was seeded per well in a 12-well Matrigel-coated plate with $2 \mathrm{ml}$ of hiPSC medium at minus day 4 . At day 0 , the medium was refreshed with RPMI/B-27 containing CHIR99021 (10 $\mu \mathrm{M}$, GSK3 inhibitor) without insulin and continued to incubate for $24 \mathrm{~h}$. The medium was replaced with RPMI/B-27 without insulin for another $48 \mathrm{~h}$. On day 3 of the differentiation ( $72 \mathrm{~h}$ after addition of CHIR99021), the medium was refreshed with RPMI/B27 containing IWP2 (5 $\mu \mathrm{M}$, Wnt inhibitor) without insulin for $48 \mathrm{~h}$, followed by RPMI/B-27 without insulin from day 5 to 7 . From day 7 , the medium was refreshed with RPMI/B-27 containing insulin every 3 days. The beating cardiomyocytes can be seen as early as on day 8 .

\section{Enriched differentiation of SAN-like cardiomyocytes from hiPSC by BMP4 (B), PD (P), and BMS (M) treatment}

Based on the GiWi protocol that it caused highly efficient pan-cardiomyocytes as shown above, different concentrations of BMP4 $(0,1.25,2.5,5 \mathrm{ng} / \mathrm{ml}), \mathrm{PD}(0,480$, $720,960 \mathrm{nM})$, and BMS $(0,1 \mu \mathrm{M})$ were added alone between day 5 and 7 after differentiation for 2 days. The qRT-PCR analysis was performed to evaluate the mRNA levels of SAN markers at day 16 to determine the optimal concentration of each compound. To investigate the synergistical effect, the cells were treated with combinations of BMP4, PD, and BMS with the optimal concentration from day 5 to 7 during the differentiation process. The markers of pacemaker cells were evaluated by analysis of qRT-PCR, immunofluorescence, and flow cytometry at day 21 . Electrophysiological characteristics were analyzed using action potential (AP) recording at day 60. The schematic protocol is shown in Fig. 4a.

\section{RNA isolation and qPCR}

Total RNA was isolated using TRIzol method (15596026, Invitrogen, USA). One microgram of total RNA was reversely transcribed in a total volume of $10 \mu \mathrm{l}$ with ReverTra Ace qPCR RT Master Mix kit (FSQ-201,
TOYOBO, Japan) following the manufacturer's instructions. The cDNA was diluted 3 times, and $1 \mu \mathrm{l}$ was used for real-time PCR in a $20-\mu \mathrm{l}$ reaction using SYBR Green Real Time PCR Mix (204143, Qiagen, Germany). The PCR conditions were $95^{\circ} \mathrm{C}$ for $2 \mathrm{~min}$, followed by $40 \mathrm{cy}$ cles of $95^{\circ} \mathrm{C}$ for $20^{\prime \prime}$ and $60^{\circ} \mathrm{C}$ for $15^{\prime \prime}$. All primers are listed in Table 1. The expression of target gene was normalized to that of GAPDH and calculated using the $2^{-\Delta \Delta \mathrm{Ct}}$ method.

\section{Immunofluorescence}

Single hiPSC cells and induced cardiomyocytes were seeded in a $\mu$-Slide 8 well $(80827$, ibidi) coated with Matrigel at the density of $2 \times 10^{4}$ per well for $48 \mathrm{~h}$. Cells were fixed with $4 \%(\mathrm{w} / \mathrm{v})$ paraformaldehyde (PFA) for $15 \mathrm{~min}$ at room temperature, permeabilized, blocked in $5 \%(\mathrm{w} / \mathrm{v}) \mathrm{BSA}$ in PBS for $30 \mathrm{~min}$ and then incubated with the following primary antibodies: anti-OCT4 antibody (\#2750, Cell Signaling Technology, USA), antiNANOG antibody (\#3580, Cell Signaling Technology, USA), anti-SSEA4 antibody (\#4755, Cell Signaling Technology, USA), anti-TRA-1-60 antibody (\#4746, Cell Signaling Technology, USA), anti-Ki67 antibody (ab15580, abcam, USA), anti-NKX2.5 antibody (ab91196, abcam, USA), anti-cTNT antibody from mouse (MS-295-P1, Thermo Fisher Scientific, USA), anti-cTNT antibody from rabbit (15513-1-AP, Proteintech, China), anti- $\alpha$ actinin antibody (A7811, Sigma, USA), anti-SHOX2 antibody (ab55740, abcam, USA), anti-TBX18 antibody (ab115262, abcam, USA), anti-COUPTFII antibody (PPH7147-00, R\&D, USA), anti-MLC2V antibody (MABT180, Sigma, USA), and anti-TBX3 antibody (ab154828, abcam, USA), followed by the following species-specific fluorescence-conjugated secondary antibodies: alexa fluor 488 labeled goat anti-rabbit IgG (A11008, Invitrogen, USA), alexa fluor 488 labeled goat anti-mouse IgG (A-11001, Invitrogen, USA), alexa fluor 594 labeled goat anti-rabbit IgG (R37177, Invitrogen, USA), and alexa fluor 594 labeled goat anti-mouse IgG (A-11005, Invitrogen, USA). The cells were then counterstained using $0.5 \mu \mathrm{g} / \mathrm{ml}$ of DAPI (4083, Cell Signaling Technology, USA) for $15 \mathrm{~min}$ at room temperature. After rinsing with PBS, the chambers were mounted and visualized under fluorescence microscopy (IX83, Olympus, Japan). Corresponding antibody isotype control, mouse IgG (ab205719, abcam, USA), rabbit IgG (ab205718, abcam, USA), mouse IgG (ab190369, abcam, USA), mouse IgG1 (\#5415, Cell Signaling Technology, USA), and mouse IgG3 (\#37988, CST, USA) were used.

\section{Flow cytometry}

The induced cardiomyocytes in 12-well plate were digested with $0.25 \%$ trypsin with $0.5 \mathrm{mM}$ EDTA into single cell suspension and washed with PBS. Cells were 
Table 1 Primer sets for qRT-PCR analysis

\begin{tabular}{|c|c|c|c|}
\hline Gene & Direction & Sequence $\left(5^{\prime}-3^{\prime}\right)$ & Product size (bp) \\
\hline \multirow[t]{2}{*}{ NANOG } & Forward & TITGTGGGCCTGAAGAAAACT & 91 \\
\hline & Reverse & AGGGCTGTCCTGAATAAGCAG & \\
\hline \multirow[t]{2}{*}{ SOX2 } & Forward & GCCGAGTGGAAACTITTGTCG & 145 \\
\hline & Reverse & GGCAGCGTGTACTTATCCTTCT & \\
\hline \multirow[t]{2}{*}{ OCT4 } & Forward & CTGGGTTGATCCTCGGACCT & 121 \\
\hline & Reverse & CCATCGGAGTTGCTCTCCA & \\
\hline \multirow[t]{2}{*}{ BRACHYURY } & Forward & CAGTGGCAGTCTCAGGTTAAGAAGGA & 122 \\
\hline & Reverse & CGCTACTGCAGGTGTGAGCAA & \\
\hline \multirow[t]{2}{*}{ MESP1 } & Forward & AGCCCAAGTGACAAGGGACAACT & 82 \\
\hline & Reverse & AAGGAACCACTTCGAAGGTGCTGA & \\
\hline \multirow[t]{2}{*}{ NKX2-5 } & Forward & CAAGTGTGCGTCTGCCTTT & 190 \\
\hline & Reverse & CAGCTCTITCTITTCGGCTCTA & \\
\hline \multirow[t]{2}{*}{ TNNT2 } & Forward & TTCACCAAAGATCTGCTCCTCGCT & 111 \\
\hline & Reverse & TTATTACTGGTGTGGAGTGGGTGTGG & \\
\hline \multirow[t]{2}{*}{$\mathrm{SHOX} 2$} & Forward & CAAAGAGGATGCGAAAGGGAT & 122 \\
\hline & Reverse & AGTGGGTCTCGTCAAAAAGCC & \\
\hline \multirow[t]{2}{*}{ TBX18 } & Forward & GACGATCTTTCTCCCATCAAGC & 124 \\
\hline & Reverse & CTATCTTCAGGCGAGTAATCTGC & \\
\hline \multirow[t]{2}{*}{ TBX3 } & Forward & CCCGGTTCCACATTGTAAGAG & 104 \\
\hline & Reverse & GTATGCAGTCACAGCGATGAAT & \\
\hline \multirow[t]{2}{*}{$\mathrm{HCN} 4$} & Forward & TGGACACCGCTATCAAAGTGG & 157 \\
\hline & Reverse & CTGCCGAACATCCTTAGGGA & \\
\hline \multirow[t]{2}{*}{ MYL7 } & Forward & AAGCCATCCTGAGTGCCTTC & 127 \\
\hline & Reverse & AACATCTGCTCCACCTCAGC & \\
\hline \multirow[t]{2}{*}{ MYL2 } & Forward & TGAGAGACACCTTTGCTGCC & 139 \\
\hline & Reverse & GGGTCCGCTCCCTTAAGTIT & \\
\hline \multirow[t]{2}{*}{ MSX2 } & Forward & CTGGTGAAGCCCTTCGAGAC & 133 \\
\hline & Reverse & ATATGTCCTCCTACTCCTGCCC & \\
\hline \multirow[t]{2}{*}{ TBX2 } & Forward & TACGCTTGTACGAGGAGCAC & 157 \\
\hline & Reverse & CACGACTTCTCCTCAGCTCG & \\
\hline \multirow[t]{2}{*}{ ISL1 } & Forward & TCCCTATGTGTTGGTTGCGG & 194 \\
\hline & Reverse & CATTTGATCCCGTACAACCTGA & \\
\hline \multirow[t]{2}{*}{ Cx30.2 } & Forward & CCGAGAACCTTGCCTTGGTA & 98 \\
\hline & Reverse & GACAGAAACCGCTGACTCCA & \\
\hline \multirow[t]{2}{*}{ Cx43 } & Forward & TACCAAACAGCAGCGGAGTT & 139 \\
\hline & Reverse & TGGGCACCACTCTITTGCTT & \\
\hline \multirow[t]{2}{*}{$C \times 40$} & Forward & AGAGTGTGAAGAAGCCCACG & 70 \\
\hline & Reverse & AACAGATGCCAAAACTTCTGCT & \\
\hline \multirow[t]{2}{*}{ HCN1 } & Forward & GCCATGCTGAGCAAGTTGAG & 178 \\
\hline & Reverse & TCAGCAGGCAAATCTCTCCA & \\
\hline \multirow[t]{2}{*}{ KCND2 } & Forward & GGGTCTTCGGCTAGCAAGTT & 91 \\
\hline & Reverse & GCACCATGTCACCATACCCTA & \\
\hline \multirow[t]{2}{*}{ KCNK2 } & Forward & TGGAACAAGACTCCTTGCTGG & 150 \\
\hline & Reverse & CTGCCGAACATCCTTAGGGA & \\
\hline
\end{tabular}


Table 1 Primer sets for qRT-PCR analysis (Continued)

\begin{tabular}{|c|c|c|c|}
\hline Gene & Direction & Sequence $\left(5^{\prime}-3^{\prime}\right)$ & Product size (bp) \\
\hline \multirow[t]{2}{*}{ KCNN4 } & Forward & CCGAGAGGCAGGCTGTTAAT & 81 \\
\hline & Reverse & AGCCGATGGTCAGGAATGTG & \\
\hline \multirow[t]{2}{*}{ KCNJ5 } & Forward & CCCACAACAGGGAGAGGTTC & 170 \\
\hline & Reverse & AGCCATAGCTGGGATGTTGTT & \\
\hline \multirow[t]{2}{*}{ KCNQ1 } & Forward & GGGCCGCGTCTACAACTTC & 169 \\
\hline & Reverse & CAGCACGATCTCCATCCAGAA & \\
\hline \multirow[t]{2}{*}{ CACNA1A } & Forward & GTCTGGGGAAGAAGTGTCCG & 151 \\
\hline & Reverse & GCTCCTCCCTTGGCAATCTT & \\
\hline \multirow[t]{2}{*}{ CACNB1 } & Forward & CCAGTGCCAAACAGAAGCAG & 181 \\
\hline & Reverse & CGAGTGATGGAGATCCTGCC & \\
\hline \multirow[t]{2}{*}{ CACNA1C } & Forward & GACGTGCTGTACTGGGTCAA & 125 \\
\hline & Reverse & AACTCTCCGCTAAGCACACC & \\
\hline \multirow[t]{2}{*}{ SCN5A } & Forward & GGAGGAGTCCAGCAAGCAG & 194 \\
\hline & Reverse & AACTGTCCTCTGGGGTCTCA & \\
\hline \multirow[t]{2}{*}{ SCN3B } & Forward & ACGCATTCTGTAGCCCAGAC & 156 \\
\hline & Reverse & CTTCCAAGGCTCTCGCCTC & \\
\hline
\end{tabular}

fixed with $4 \%$ formaldehyde for $10 \mathrm{~min}$ at room temperature and chilled on ice for $1 \mathrm{~min}$. Permeabilization was performed by adding one tenth of ice-cold $100 \%$ methanol slowly to the pre-chilled cells and continued to incubate on ice for $30 \mathrm{~min}$. Cells were then blocked with blocking buffer (0.5\% BSA in PBS) for $10 \mathrm{~min}$; incubated with the following primary antibodies: anti-OCT4 antibody (\#2750, Cell Signaling Technology, USA), antiNANOG antibody (\#3580, Cell Signaling Technology, USA), anti-SSEA4 antibody (\#4755, Cell Signaling Technology, USA), anti-TRA-1-60 antibody (\#4746, Cell Signaling Technology, USA), anti-Ki67 antibody (ab15580, abcam, USA), anti-cTNT antibody (MS-295-P1, Thermo Fisher Scientific, USA), anti-NKX2-5 antibody (ab91196, abcam, USA), and anti-SHOX2 antibody (ab55740, abcam, USA) for $1 \mathrm{~h}$ at room temperature; then washed with PBS; and followed by incubation with the corresponding speciesspecific fluorescence-conjugated secondary antibodies: alexa fluor 488 labeled goat anti-mouse IgG (A-11029, Invitrogen, USA), alexa fluor 488 labeled goat anti-rabbit IgG (A11034, Invitrogen, USA), alexa fluor 647 labeled goat antimouse IgG (A-21235, Invitrogen, USA), and alexa fluor 647 labeled goat anti-rabbit IgG (A-32733, Invitrogen, USA) for $30 \mathrm{~min}$ at room temperature. Cells were analyzed using a flow cytometry machine (651155, BD FACS Verse, BD Bioscience, USA) according to the manufacturer's protocol.

\section{Action potential (AP) recording}

AP recording was performed following El-Battrawy et al.'s protocol with some modifications [20]. Briefly, on 60 days after cardiomyocyte differentiation, induced cardiomyocytes were dissociated into single cell suspension by 30 min type
I collagenase $(2 \mathrm{mg} / \mathrm{ml})$ followed by $3 \mathrm{~min}$ trypsin $(0.25 \%)$ without EDTA. $1 \times 10^{4}$ cells were seeded into a $3.5-\mathrm{cm}$ dish containing a lysine-treated glass coverslip and incubated for 3 days. AP was recorded using the whole cell patch clamp electrophysiology method. Briefly, the adherent cells on the coverslip were placed in the recording chamber and perfused with bath solution containing $140 \mathrm{mM} \mathrm{NaCl}, 1 \mathrm{mM}$ $\mathrm{MgCl}_{2}, 5 \mathrm{mM} \mathrm{KCl}, 1.8 \mathrm{mM} \mathrm{CaCl}, 5 \mathrm{mM}$ 4-(2-hydroxyethyl)-1-piperazineethanesulfonic acid (HEPES), and 10 $\mathrm{mM}$ glucose (the $\mathrm{pH}$ was adjusted to 7.40 , and the osmolality to $301 \pm 3 \mathrm{mOsm}$, respectively). The patch pipettes were pulled from borosilicate glass capillaries (7-000-0650-LHC, Drummond, USA) by a horizontal puller (PC100, NARI SHIGE, Japan) and had resistances of 1.5-3 M . Pipette solution consisted of $110 \mathrm{mM} \mathrm{K}$-gluconate, $20 \mathrm{mM} \mathrm{KCl,} 1$ $\mathrm{mM} \mathrm{CaCl} 2,1 \mathrm{mM} \mathrm{MgCl}_{2}, 10 \mathrm{mM}$ HEPES, $5 \mathrm{mM}$ ethylene glycol tetraacetic acidpotassium chloride (EGTA-KOH), 5 $\mathrm{mM}$ ATP- $\mathrm{Mg}^{2+}$, and $5 \mathrm{mM}$ Na-phosphocreatine. The $\mathrm{pH}$ was adjusted to 7.2 by $\mathrm{KOH}$, and the osmolality to $290 \pm 3$ mOsm. A Multiclamp 700B amplifier was used to record APs, and data were analyzed using a custom software.

\section{Statistical analysis}

Experimental data are presented as "mean \pm SD" with at least three repeats. Comparisons between multiple groups were performed using one-way analysis of variance (one-way ANOVA), with $p<0.05$ considered as statistically significant.

\section{Results}

\section{Characterization of hiPSC}

hiPSC and human embryonic stem cell (hESC) were purchased from OSINGLAY BIO company. To confirm 
the authenticity of the hiPSC, the molecular signature of hiPSC was validated by both qRT-PCR and immunofluorescence (IF). The qRT-PCR results showed that the hiPSC expressed stem cell-specific markers OCT3/4, SOX2, and NANOG at similar levels as hESC which serves as a positive control. In contrast, these markers were non-detectable in the terminal differentiated human terminal differentiated fibroblast cells $(B j)$ as a negative control (Fig. 1a). The IF results showed that the hiPSC was proliferative (Ki67 positive) (Fig. 1b) and heavily stained for NANOG, OCT4, SSEA4, and TRA-160 , which were localized in the nuclei (for NANOG and OCT4) and plasma membrane (for SSEA4 and TRA-160), respectively (Fig. 1c-f). This was further confirmed using flow cytometry analysis (Fig. 1g, h). These results demonstrate that the hiPSC possesses their pluripotency and self-renewal capability.

\section{Identification of hiPSC-induced cardiomyocytes}

Cardiomyocytes were induced by the widely used GiWi protocol originally proposed by Lian et al. [19], where the induction process was temporally initiated using GSK3 inhibitor (Gi) and followed by temporal inhibition of the Wnt signaling pathway using Wnt inhibitor (Wi). To optimize the induction protocol, different concentrations of the Gi, CHIR were added on day 0 of the differentiation and continued to incubate for $24 \mathrm{~h}$, and the markers of mesoendoderm (BRACHYURY) and cardiac mesoderm (MESP1) were evaluated on day 1 and day 3 using qRT-PCR. CHIR dose-dependently increased the

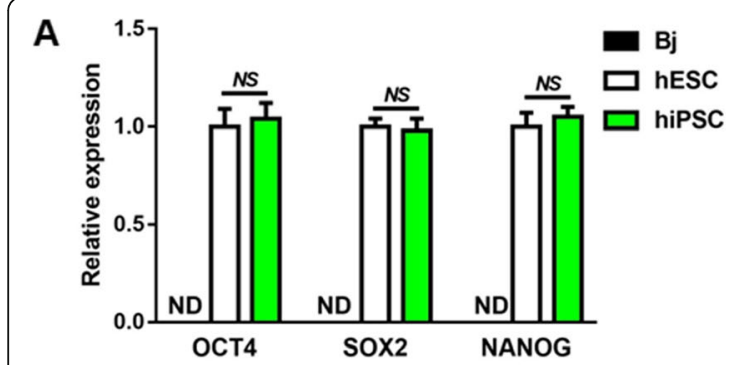

\section{B}

E

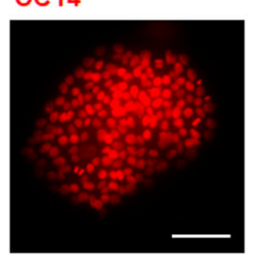

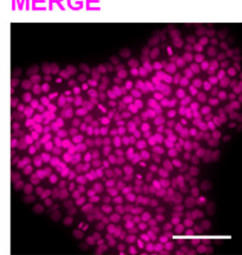

DAPI

DAPI
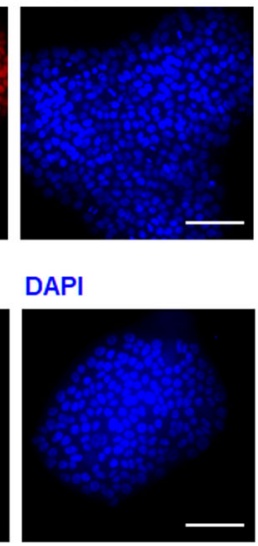

MERGE

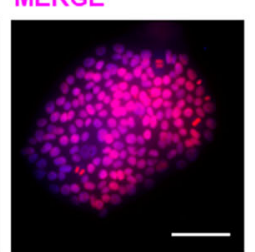

KI67

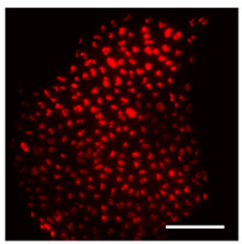

DAPI

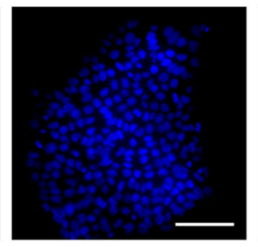

DAPI

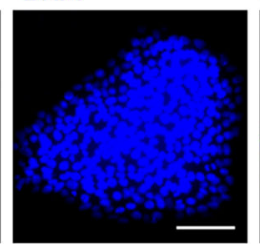

DAPI

F

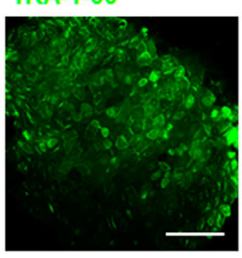

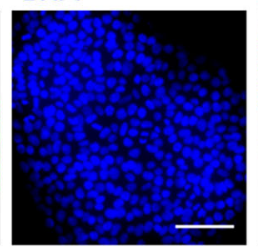

MERGE

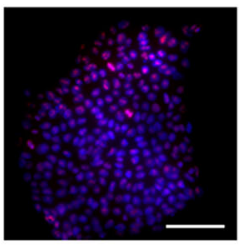

MERGE

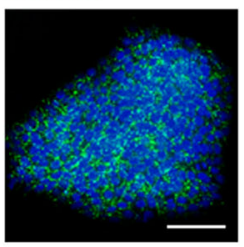

MERGE

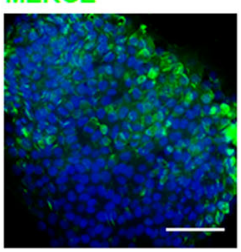

G

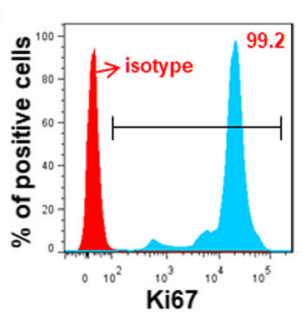

H

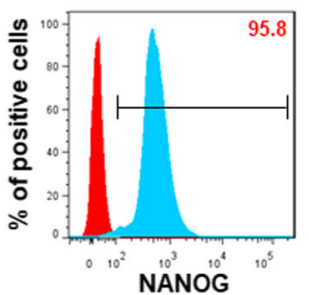

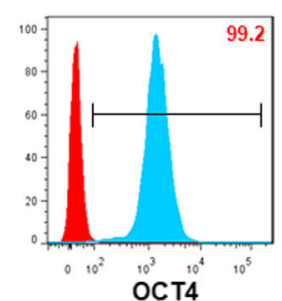

OCT4
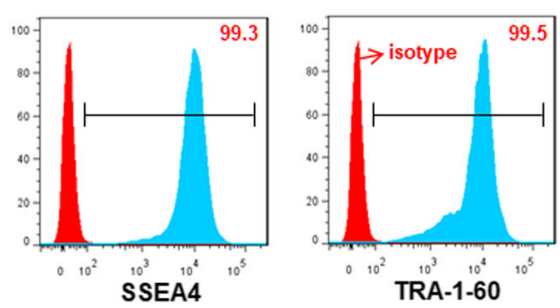

Fig. 1 Characterization of the hiPSC. a hiPSC expressed high levels of the pluoripotency marker genes, OCT3/4, SOX2, and NANOG, which were comparable to hESC, a human embryonic stem cell ( $t$ test; NS, not significant; ND, not detectable; $n=3$ ). b Ki67, pluoripotency markers of hiPSC were identified using IF method. c-e Pluoripotency markers of hiPSC (NANOG, OCT4, SSEA4, and TRA-1-60) were confirmed by

immunoflorescence (IF) assay. $\mathbf{g}, \mathbf{h}$ Representative flow cytometry analysis further confirmed the pluoripotency of the hiPSC. Scale bars, 100 um (×400). Expression values of all PCR analyses were normalized to the housekeeping gene GAPDH. Data are presented as "mean \pm SD" 
expression of both BRACHYURY (Fig. 2a) and MESP1 (Fig. 2b) on day 1 , with CHIR at $10 \mu \mathrm{M}$ reached the highest level. Similar concentration-response curves were observed on day 3 for both markers (Fig. 2a, b). However, the overall levels of BRACHYURY (Fig. 2a) were significantly decreased, while the levels of MESP1 (Fig. 2b) were significantly increased, at different concentrations of CHIR compared to those levels on day 1 . These observations suggest fate conversion from mesoendoderm into cardiac mesoderm. The successful induction of cardiomyocytes at day 21 from differentiation was confirmed by both IF, showing positive staining for cardiomyocyte markers, cTNT and $\alpha$-actinin (Fig. 2c), and flow cytometry, showing that around 85\% of the induced cardiomyocytes expressed the cardiac sarcomere proteins, cTNT (Fig. 2d). Quite high percent of beating cardiomyocytes was observed at day 21 as shown in SUPPL-Video 1. This is in sharp contrast with the spontaneously differentiated cells without GiWi treatment in SUPPL-Video 2. These observations further confirmed our optimized differentiation procedure.

\section{BMP, RA, and FGF signaling pathways contribute differently to the differentiation of cardiomyocytes toward SAN-like cells}

Although it is generally agreed that ventricular, atrial, and SAN-like cardiomyocytes are derived from different progenitor cells emerging as early as mesoderm stage, and different signaling pathways contribute differently to the directional differentiation toward ventricular, atrial, and SAN-like cardiomyocytes, the timing of manipulation of the signaling pathways remains controversial. To determine the timing of manipulation, we evaluated the temporal expression of cardiac mesoderm marker (MESP1), cardiac progenitor marker

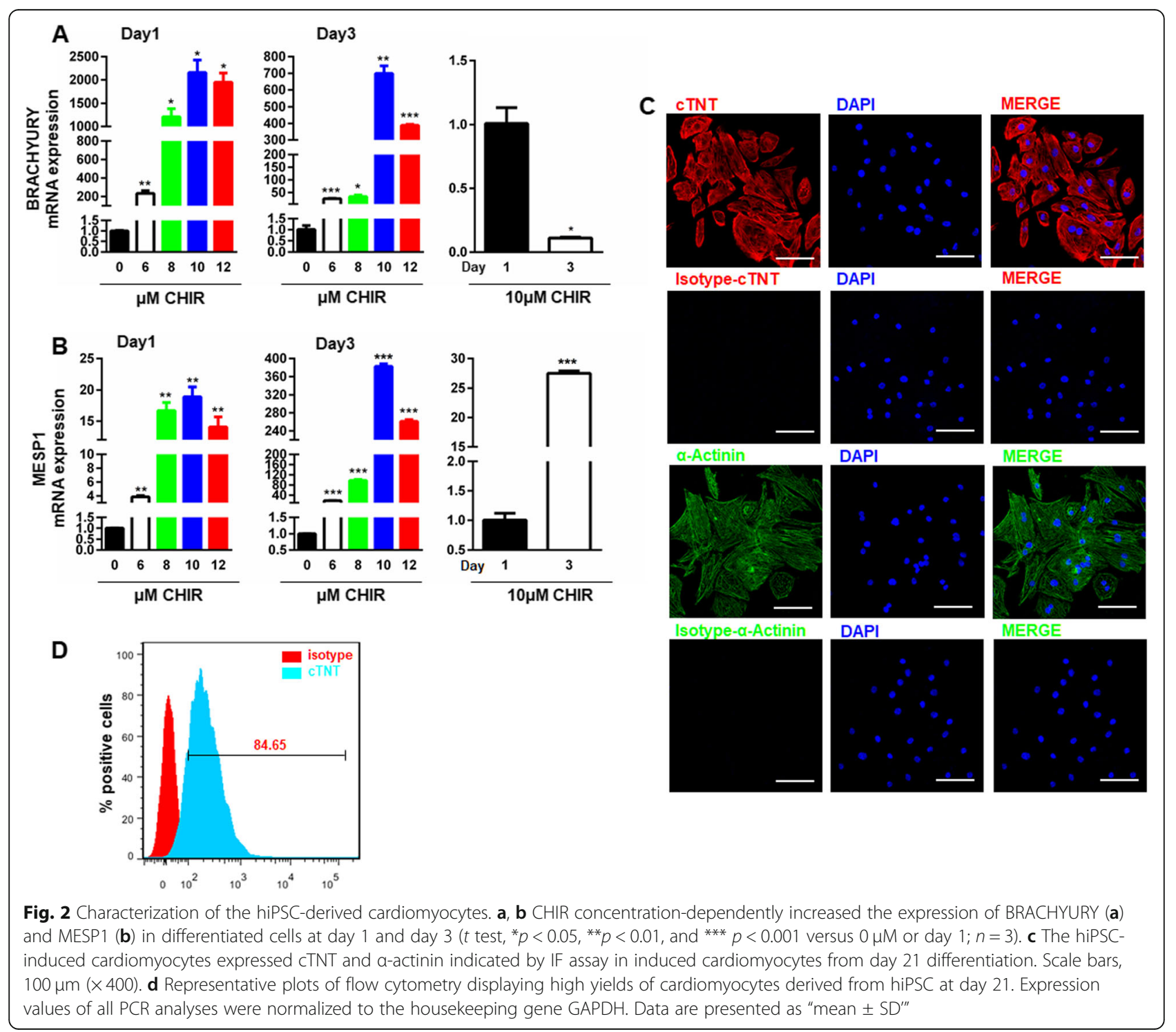


(NKX2-5), and pan-cardiomyoyte marker (TNNT2) by qRTPCR. As shown in Fig. 3, with the progression of cardiomyocyte differentiation, the expression of MESP1 reached the highest level on day 5 and sharply dropped on days 7 and 10, suggesting that day 5 is equivalent to the developmental cardiac mesoderm stage. In contrast, NKX2-5 expression dramatically increased and reached the highest level on day 7 equivalent to the cardiac progenitor stage, and TNNT2 were
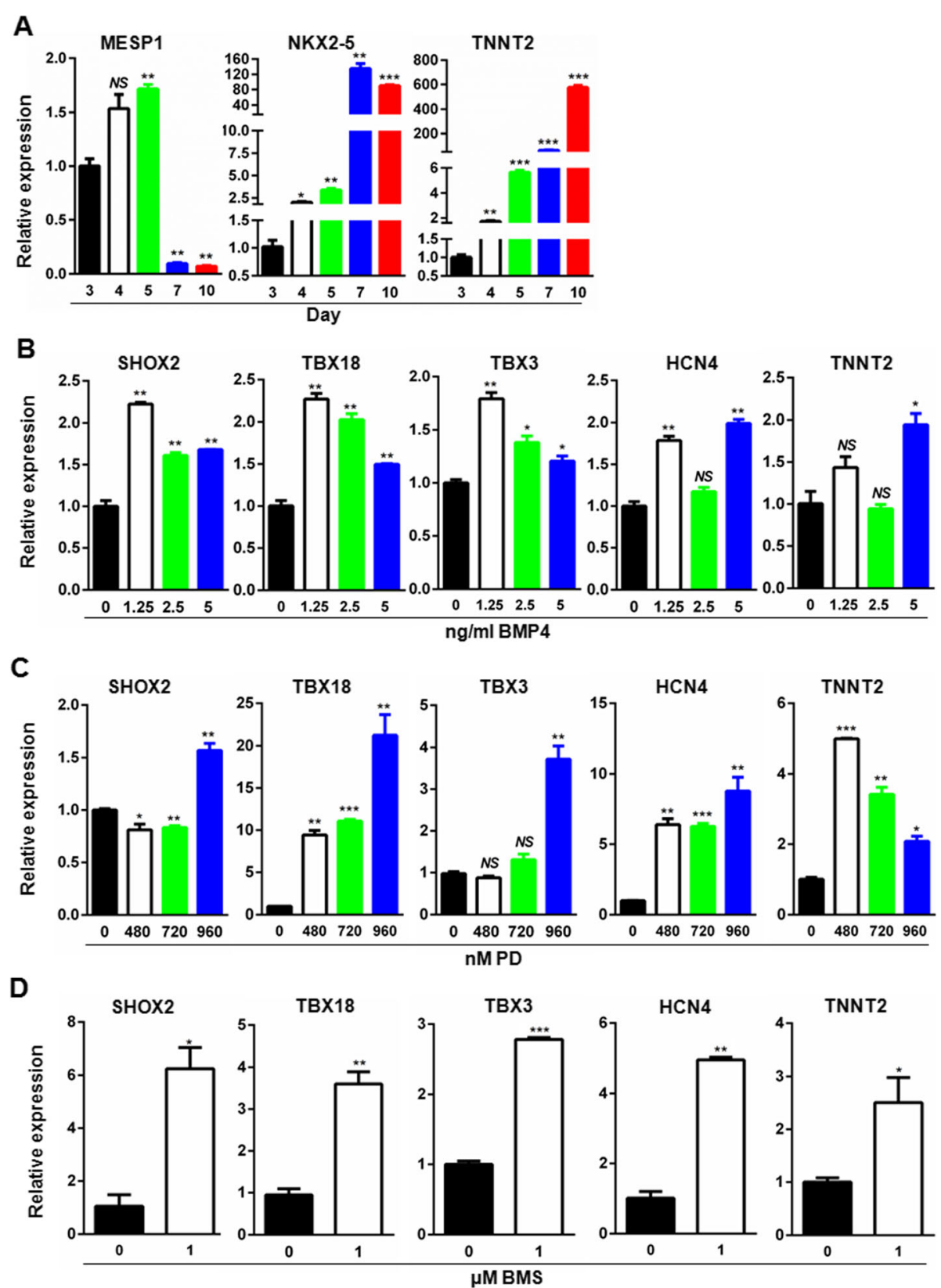

Fig. 3 Optimization of the timing and concentration of small molecule chemicals targeting FGF, RA, and BMP signaling pathways for enriched SANLC differentiation. a The expression of cardiac mesoderm marker (MESP1), cardiac progenitor marker (NKX2-5), and cardiomyocyte marker (TNNT2) at days 3, 4, 5, 7, and 10, respectively, by qRT-PCR ( $t$ test, ${ }^{*} p<0.05,{ }^{* *} p<0.01$, and ${ }^{* * *} p<0.001$ versus day $3 ; n=3$ ). $\mathbf{b}$ The expression of SHOX2, TBX18, TBX3, HCN4, and TNNT2 was evaluated by qRT-PCR at day 16 after the differentiation of hiPSC-induced cardiomyocytes was treated with BMP4 at the indicated concentrations at day 5-7 ( $t$ test, ${ }^{*} p<0.05,{ }^{* *} p<0.01$, and NS, not significant versus $0 \mu \mathrm{M} ; n=3$ ). c The expression of SHOX2, TBX18, TBX3, HCN4, and TNNT2 was analyzed by qRT-PCR at day 16 of the differentiation after the induced cardiomyocytes were treated with PD at the indicated concentrations at day $5-7$ ( $t$ test, ${ }^{*} p<0.05,{ }^{* *} p<0.01,{ }^{* * *} p<0.001$, and NS, not significant versus $0 \mu \mathrm{M} ; n=3$ ).d The expression of SHOX2, TBX18, TBX3, HCN4, and TNNT2 was analyzed by qRT-PCR at day 16 of the differentiation after the induced cardiomyocytes were treated with BMS at the indicated concentrations at day $5-7$ ( $t$ test, ${ }^{*} p<0.05$ and ${ }^{* *} p<0.01$ versus $0 \mu \mathrm{M} ; n=3$ ). Expression values of all PCR analyses were normalized to the housekeeping gene GAPDH. Data are presented as "mean \pm SD" 
upregulated in a time-dependent manner (Fig. 3a). These results suggest that it is from day 5 to day 7 during which cells experienced fate conversion from the cardiac mesoderm to the progenitor. Given that the SANLC development originates as early as cardiac mesoderm, the optimal time window of $48 \mathrm{~h}$ between day 5 and day 7 was determined to manipulate the SANLC-enriched differentiation process using different signaling pathway modulators. The schematic illustration of SANLC-enriched differentiation protocol based on the GiWi method was shown in Fig. 4a.

BMP4 has been shown to increase the proportion of SANLC from hiPSC via activating the bone morphogenetic protein (BMP) signaling pathway. To optimize the concentration of BMP4 on the differentiation of hiPSC to the SANLPC, hiPSC was stimulated with different concentrations of BMP4 for 2 days and the expression of SAN markers SHOX2, TBX18, TBX3, and HCN4 were analyzed at day 16. Consistent with a previous report that lower concentration of BMP4 could increase the proportion of SANL PC from hiPSC, BMP4 at $1.25 \mathrm{ng} / \mathrm{ml}$ maximumly increased the expression of the SAN markers, SHOX2, TBX18, TBX3, and HCN4, but not the pan-cardiomyocyte marker TNNT2 that showed no response at lower concentrations and reached statistical significance only at $5 \mathrm{ng} / \mathrm{ml}$ (Fig. 3b). Interestingly, further increased concentrations of BMP4 tended to concentration-dependently weaken the effect of BMP4 on the expression of TBX18, SHOX2, and TBX3
(Fig. 3b). $1.25 \mathrm{ng} / \mathrm{ml}$ per milliliter was therefore chosen as the optimal concentration for BPM4.

NKX2-5-mediated fibroblast growth factor (FGF) signaling has been proposed to contribute to the differentiation of hiPSC to ventricular cardiomyocytes [13, 14]. To optimize the inhibition of FGF signaling pathway on the SANLPC generation, the FGF inhibitor PD was added in the hiPSC and the expression of SHOX2, TBX18, TBX3, HCN4, and TNNT2 were analyzed at day 16 . The result showed that PD concentration-dependently upregulated all the SANLPC markers. As to TNNT2, PD at $450 \mathrm{nM}$ significantly upregulated its expression, which was inhibited by increasing concentrations of $\mathrm{PD}$ in a concentration-dependent manner (Fig. 3c). Taken together, these observations indicate that PD at concentration of $960 \mathrm{nM}$ is optimal.

Finally, the effect of retinoid acid (RA) signaling on SANL PC differentiation was also investigated at day 16. Treatment of hiPSC with $1 \mu \mathrm{M}$ of the RA signaling inhibitor BMS significantly increased the expression of the markers of pacemaker cells HOX2, TBX18, TBX3, and HCN4 and the pancardiomyocyte marker TNNT2 (Fig. 3d).

\section{Combined modulation of the three signaling pathways} synergistically promoted the differentiation of hiPSC to SANLC Having optimized the conditions of manipulating bone morphogenetic protein (BMP), fibroblast growth factor (FGF), and retinoid acid (RA) signaling pathways

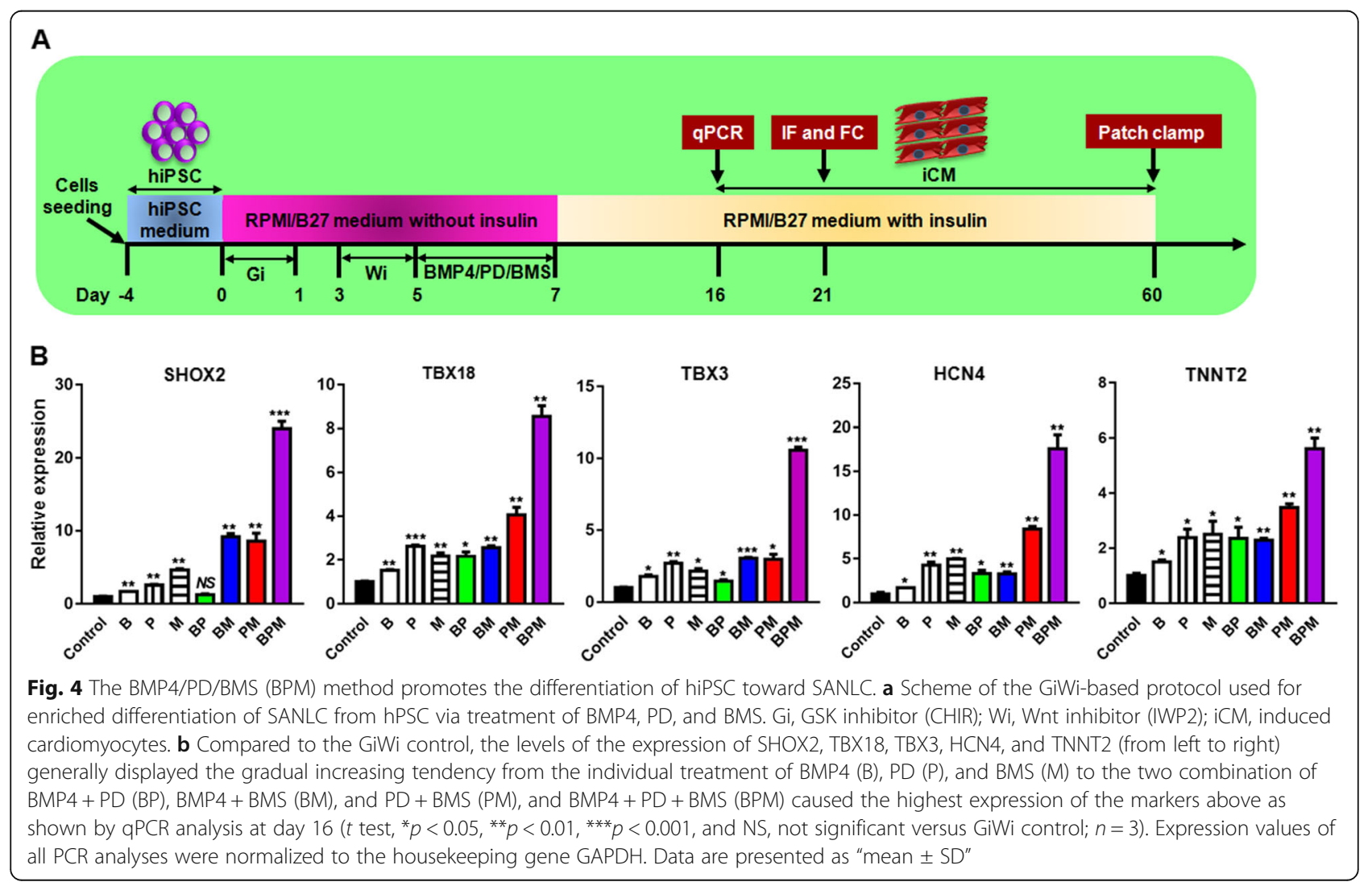


individually on the differentiation of hiPSC to SANLC, we tested whether combined usage of the three modulators BMP4, PD, and BMS (they are abbreviated as B, P, and $M$ correspondingly in Fig. $4 \mathrm{~b}$ ) has any synergistical effect. The scheme of SANLC-enriched differentiation protocol based on GiWi method is shown in Fig. 4a, where the detailed information regarding time points for the treatment and testing analysis was included. As shown in Fig. 4b, BMP4 + PD, BMP4 + BMS, PD + BMS, and BMP4 + PD + BMS (they are abbreviated as BP, BM, PM, and BPM correspondingly in Fig. 4b) combinations all significantly increased the expression of the pacemaker cell markers SHOX2, TBX18, TBX3, and HCN4 and the pan-cardiomyocyte marker TNNT2 compared to those of the GiWi protocol. Among the four combinations, BPM had the best induction efficiency (Fig. 4).

\section{The BMP4 + PD + BMS (BPM) induction protocol leads to} biased differentiation of hiPSC to SANLC

Since the BMP4 + PD + BMS (BPM) induction protocol most effectively increased the gene expression of the markers of pacemaker cells, we next evaluated the expression of the aforementioned markers at protein level using IF and flow cytometry on day 21 . IF results showed that most nuclei were positive for transcription factors SHOX2, TBX3, and TBX18 (Fig. 5a-c). Likewise, flow cytometry data showed that BPM induction protocol generated a higher percentage of SANLC (defined by cTNT $\left.{ }^{+} / \mathrm{NKX}^{-} .5^{-}\right)(55.1 \pm 5 \%)$ compared to the traditional GiWi induction protocol $(34.1 \pm 2 \%)$ (Fig. 5d). When SANLC was defined by $\mathrm{CTNT}^{+} / \mathrm{SHOX}^{+}$, the BPM protocol again generated a higher percentage of SANLC $(44.5 \pm 2 \%$ in the BPM versus $22.4 \pm 5 \%$ in the GiWi) (Fig. 5e). The results of Fig. 5d, e were further confirmed by IF analysis of double staining for cTNT/NKX2.5 and cTNT/SHOX2 as shown in SUPPL-Fig. 1. It seemed that the SAN cells identified by $\mathrm{cTNT}^{+} / \mathrm{NKX} 2.5^{-}$and $\mathrm{cTNT}^{+} / \mathrm{SHOX2}^{+}$were more likely to display the similar morphological characteristics indicated by elongated or spindle-like shapes that are consistent to the previous report.

It may be inferred that enriched SANLC population by BPM resulted in the reduced ratio of other two cardiomyocyte subtypes: ventricular and atrial cells. To further evaluate the effect of BPM, the expression of ventricular and atrial related markers was tested using qPCR analysis at day 16. Consistent with our hypothesis, the levels of both atrial (MYL7 and COUPTFII) (SUPPL-Fig. 2A) and ventricular (MYL2 and NKX2.5) (SUPPL-Fig. 2B) markers were significantly decreased in the BPM group. These results were further supported by IF analysis of COUPTFII and MLC2V whose coding gene is MYL2 (SUPPL-Fig. 2C). The markers of another automatic cell subtype atrioventricular node (AVN) cell were investigated using qPCR analysis at day 16. The results showed that the expression of SAN markers MSX2 and TBX2 was reduced by BPM (SUPPL-Fig. 3).

\section{SANLC generated by the BPM protocol displays the typical} electrophysiological characteristics of pacemaker cells To evaluate the automaticity of the SANLC, cells were observed under microscopy and the beating rate was recorded. The representative movies of beating cardiomyocytes shown
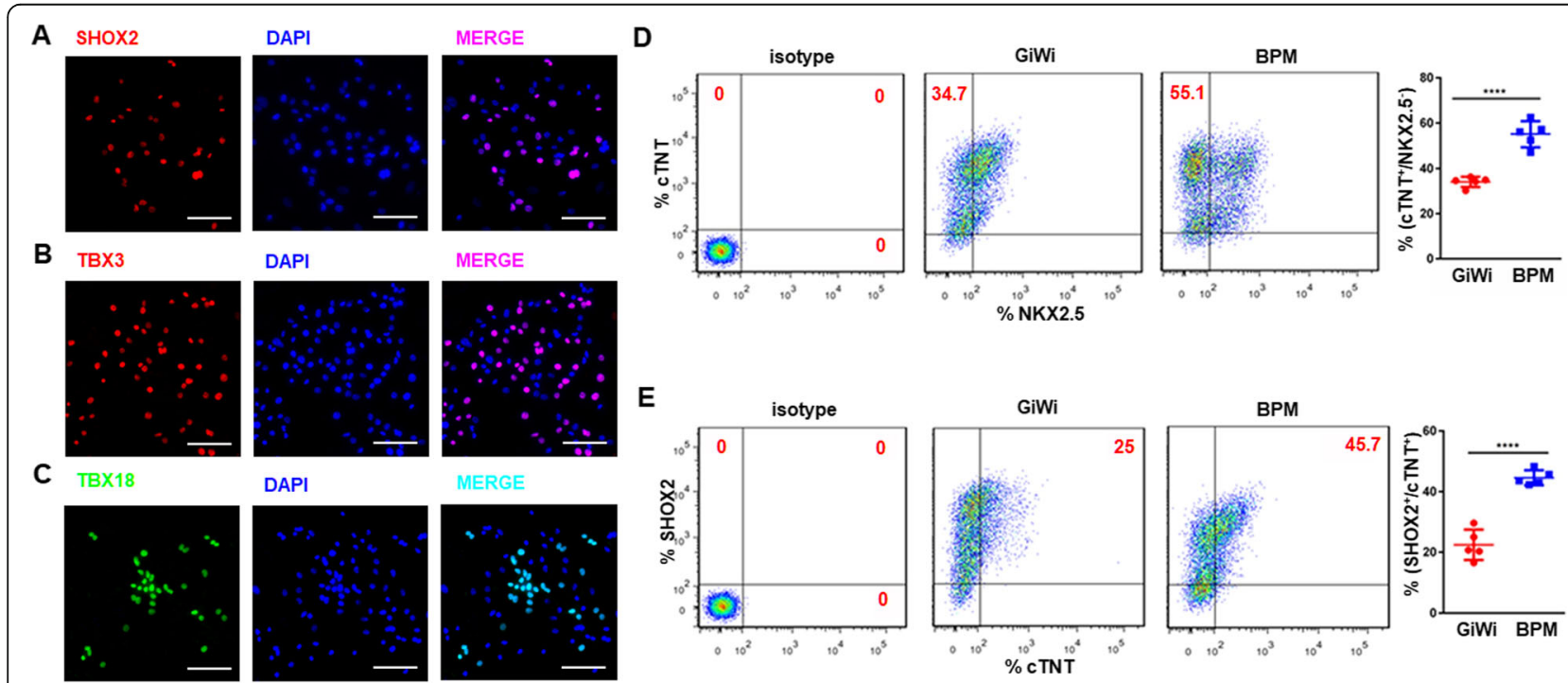

Fig. 5 Validation of the enriched differentiation of SANLC by the BMP4/PD/BMS (BPM). a-c Representative IF analysis showed that SANLCS expressed SAN-specific transcription factor, SHOX2, TBX3, and TBX18. Scale bars, $100 \mu \mathrm{m}(\times 400)$. d Representative flow cytometry analysis showed that BPM significantly increased the percentage of CTNT ${ }^{+} / \mathrm{NKX} 2.5^{-}$cells as compared to the GiWi group ( $t$ test, ${ }^{* * *} p<0.001$ versus GiWi control; $n=5)$. e Representative flow cytometry analysis showed that BPM significantly increased the percentage of $\mathrm{CTNT}^{+} / \mathrm{SHOX}^{+}$cells as compared to the GiWi group ( $t$ test, ${ }^{* * *} p<0.001$ versus GiWi control; $n=5$ ). Data are presented as "mean $\pm \mathrm{SD}^{\prime \prime}$ 
in SUPPL-Video 3 (GiWi group) and SUPPL-Video 4 (BPM group) displayed that the beating rate of SANLC was significantly higher in the BPM protocol $(n=6)$ than in the GiWi protocol $(n=6)$ (Fig. 6a). Since hyperpolarization-activated cyclic nucleotide-gated channel (HCN4) contributes the most to the automaticity in SAN, inhibition of the HCN4 would decrease the automaticity of the SANLC. Indeed, inhibition of the $\mathrm{HCN} 4$ using zatebradine hydrochloride significantly decreased the beating rate of SANLC from the BPM protocol $(n=7)$ than the GiWi protocol $(n=6)$, suggesting a higher percentage of SANLC in the BPM protocol than in the GiWi protocol (Fig. 6b). We then analyzed the AP using whole cell patch clamp technique 60 days after the differentiation. Based on the morphology of AP, ratio of AP duration at $90 \%$ repolarization (APD90) to APD50, upstroke velocity, and maximum diastolic potential, SANLC from both groups contained ventricle-like, atrial-like, and pacemaker-like cells (Fig. 6c). However, the percentage of pacemaker-like cells was significantly higher in the BPM protocol than in the GiWi protocol (Fig. 6c).

Different expression profiles of the ion channels could account for the different electrophysiological properties among SAN, ventricular, and atrial cell subtypes. To further confirm the SANLC-biased effect of BPM, a series of ion channel genes were checked by qPCR at day 16 . In addition to the SANLC markers (SHOX2, TBX18, TBX3, and HCN4) (Figs. 4 and 5), two other SANLC markers ISL1 and HCN1 displayed increased expression levels by BPM treatment (SUPPL-Fig. 4A). The qPCR analysis of potassium ion channels showed that BPM increased the SANLC-specific channel genes (KCND2, $\mathrm{KCNK} 2$, and KCNN4) but reduced the levels of ventricle specifically expressed gene KCNQ1 and atria specifically expressed gene KCNJ5 (SUPPL-Fig. 4B). Regarding the calcium ion channels, the expression of SANLC channel genes (CACNA1A and CACNB1) were upregulated by $\mathrm{BPM}$, whereas a ventricular ion channel marker $\mathrm{CACN}$ A1C was reduced (SUPPL-Fig. 4C). Similar result of sodium ion channel was obtained, as evidenced by increased pacemaker cell specifically expressed gene SCN3B and decreased ventricle specifically expressed gene SCN5A (SUPPL-Fig. 4D). In addition, the result of testing the gap junction channel related genes showed that BPM treatment caused remarkable induction of SANLC-specific CX30.2. However, expression for both ventricular CX43 and atrial CX40 was decreased (SUPPL-Fig. 4D).

\section{Discussion}

In this study, using the system of cardiomyocyte differentiation from hPSC by temporally manipulating the canonical Wnt signaling pathway for cardiac development stimulation, we discovered that SANLCs can be significantly enriched by simultaneous manipulation of BMP, FGF, and RA signaling pathways. These biasedly enriched SANLCs express SAN-specific markers that are sensitive to HCN4 channel blocker, and possess the electrophysiological property of native SAN cardiomyocytes.

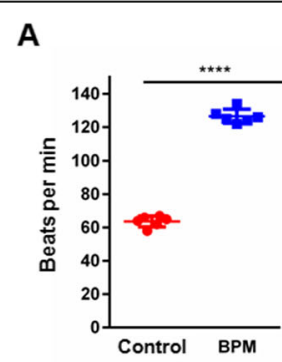

C

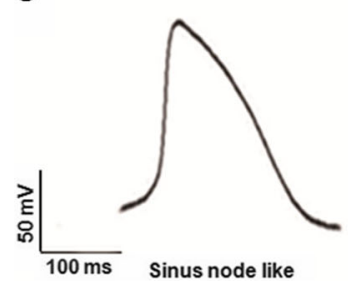

$\mathbf{B}$
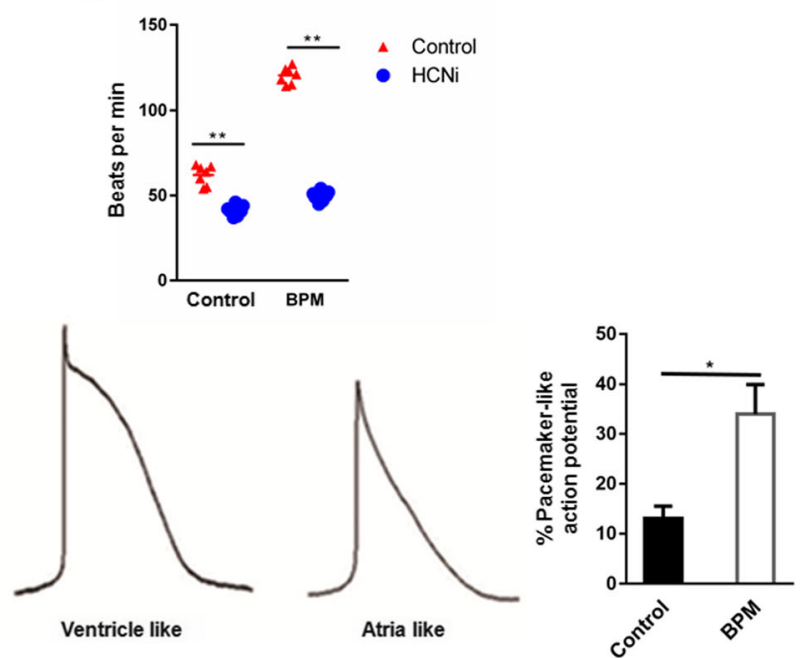

Fig. 6 SANLCs induced by the BMP4/PD/BMS (BPM) possess typical electrophysiological characteristics of SAN. a Spontaneous beating frequency of SANLCs was significantly higher in the BPM $(n=6)$ than in the GiWi groups $(n=6)\left(t\right.$ test, ${ }^{* * *} p<0.001$ versus GiWi control followed by Bonferroni's post hoc test). $\mathbf{b} \mathrm{HCN} 4$ channel inhibitor treatment caused significantly decreased beating rate in the BPM $(n=7)$ compared to the GiWi groups $(n=7)$ ( $t$ test, ${ }^{* *} p<0.01$ versus GiWi control followed by Bonferroni's post hoc test). c Representative ventricule-like, atria-like, and sinus node-like action potential (AP) curves were recorded by whole cell patch clamp (left panel). The percentage of cells with pacemaker-like AP was remarkably increased in the BPM compared to the GiWi group (6/18 versus 2/15, BPM versus GiWi) ( $t$ test, ${ }^{* * *} p<0.001$ versus GiWi control). Data are presented as "mean \pm SD" 
Biased differentiation of cardiomyocytes to SANLCs could be achieved by either manipulating the expression of SAN-specific transcription factors or using pathwayspecific activators/inhibitors. For example, TBX18 is restrictedly expressed in SAN where it promotes the development of pacemaker cardiomyocytes and at the same time prevents the activation of genes leading to chamber cardiomyocyte development. Accordingly, forced expression of TBX18 in hiPSC resulted in increased differentiation to SAN-like cardiomyocytes [21]. In addition, overexpression of TBX18 could convert the human working cardiomyocytes, adult rat bone mesenchymal stem cell, and adipose-derived stem cells into functional SAN-like cardiomyocytes [22, 23]. However, these methods of genetic manipulation for SAN regeneration are not desirable in the future clinical applications.

Previous investigations have demonstrated that SANlike cells could be induced from different cell types by gene modification-dependent strategy. In our study, we established a gene-free and chemical-induced method for highly efficient differentiation of SAN-like cells from hiPSC, which may be more amendable in future clinical use. In a recent study, Protze et al. introduced a genefree method for SAN cell induction from hPSC [24]. Based on the system of embryonic body-based cardiomyocyte differentiation, they showed that modulating the BMP and RA signaling pathway enables highly efficient $\mathrm{NKX}^{2} 5^{-} / \mathrm{cTNT}^{+}$SAN cell induction $(55 \pm 5 \%)$ indicated by flow cytometry results which is similar to our results $(55.1 \pm 5 \%)$. It was suggested that transgeneindependent method may serve as a faster, simpler, and higher efficient strategy for SAN cell generation.

BMP signaling has been reported to participate in the induction of cardiac mesoderm and formation of the first heart field [17, 25]. Low concentration of recombinant BMP4 could induce cardiac mesoderm specification from hPSC and, more importantly, increase the proportion of SAN-like progenitor cells in a time window- and concentration-sensitive manner [8]. In line with these observations, our investigation showed that treatment of hiPSC with low concentration of BMP4 during the cardiac mesodermal stage increased the yields of SAN-like cardiomyocytes. However, by increasing the dosage of BMP4, it weakened the expression of SAN-specific markers.

FGF signaling is indispensable for promoting and maintaining the characteristics of ventricle during the early stage of heart development. Activation of FGF signaling sustains ventricular development in the early stage by maintaining the number and the electrical characteristics of ventricular cardiomyocytes, while inhibition of FGF signaling results in a gradual accumulation of atrial cardiomyocytes, and a concomitant decreasing number of ventricular cardiomyocytes [26]. Further studies showed that NKX2-5 is the downstream regulator of the FGF signaling [13, 14]. Indeed, our study found that inhibition of FGF signaling downregulated the expression of ventricle-specific makers including NKX25, while enhanced the expression of SAN makers.

RA signaling is not only essential for normal heart development but also involved in the differentiation and specification of atrial cardiomyocytes. Previous studies have shown that activation of RA signaling in hiPSC differentiation is sufficient to generate cardiomyocytes displaying both electrophysiological characteristics and gene expression profile of atrial cardiomyocytes. However, study by Protze et al. showed that activation of RA signaling increases the expression of some markers of SAN and does not affect the induction efficiency in hiPSC differentiation [24]. Our study showed that antagonizing RA signaling significantly increased the expression of SAN-specific markers SHOX2, TBX3, and HCN4 that promoted SAN differentiation. Considering the discovery by Protze et al. that timely activation of and the optimal activation of RA signaling promote the maturation of SAN, we tend to believe that the biological effect of RA signaling is not just involved in atrial development but also in SAN differentiation, which warrants future investigations. It is worth mentioning that the effect of RA in the SAN development may be in the spatiotemporal-dependent manner. In fact, Protze et al. showed that the effective time window of RA treatment enhancing pacemaker characteristics is $3-7$ days in the hiPSC differentiation [24].

The effect of signal pathway modulators has been generally considered to be sensitive to the concentrations. For example, study by Protze et al. showed that BMP4 treatment at the low concentration range $(1.25-5 \mathrm{ng} / \mathrm{ml})$ could enhance the differentiation of SANLC from hiPSC [24]. In contrast, higher concentrations significantly inhibited the whole cardiomyocyte differentiation including SANLC [24]. Similarly, our study showed that the induction effect of BMP4 on the expression of SANLC markers gradually waned when increasing the concentrations (1.25, 2.5, $5 \mathrm{ng} / \mathrm{ml}$ ) (Fig. 3b). One of the interesting observations is that the three combinations (BMP4 + PD + BMS) but not two combinations (BMP4 + $\mathrm{PD}, \mathrm{BMP} 4+\mathrm{BMS}, \mathrm{PD}+\mathrm{BMS})$ demonstrated additional effect on the expression of SANLC markers when compared to the individual treatment (BMP4, PD, BMS). This suggests that the cardiomyocyte differentiation from hiPSC simulating the fetal heart development is a very complex process in a highly coordinated spatiotemporal manner, in which the terminal effect of combing different signaling pathways modulators was determined by a number of factors, such as concentration and time window.

\section{Study limitation}

The present study has several limitations that are worth mentioning. A new efficient transgene-independent 
differentiation protocol for generating SANLC from hiPSC via combined modulating BMP, FGF, and RA signaling was not completely verified at the protein levels when compared to a broader coverage of the mRNA levels. However, several SANLC-specific markers (SHOX2, TBX18, and TBX3) in BPM-caused cells were confirmed by immunocytochemistry (Fig. 5a-c). The transcription factors coded by these three marker genes have generally been thought to play the most important role in SAN cell specification, patterning, and maturation $[12,24]$. The mostly well-recognized double staining for cTNT/NKX2.5 was used to further verify the SANLC population induced by BPM treatment using flow cytometry and immunocytochemistry at the protein levles (Fig. 5d, SUPPL-Fig. 1) [24, 27], and it was further consolidated by another identification standard of SHOX2/cTNT (Fig. 5e, SUPPL-Fig. 1). Our in vitro studies demonstrated that BPM-enriched cells display the basic characteristics of SAN cells including higher beating rate, typical action potential, and sensitivity to the SAN cell-specific HCN4 channel inhibitor. However, the abilities of BPM-enriched cells to engraft within the heart and to drive the working cardiomyocytes at physiological rates in vivo animal model remain to be addressed. Our study showed that sequential modulation of Wnt signaling using the GiWi method was highly efficient enough to induce the pan-cardiomyocyte differentiation from hiPSC. However, we did not investigate the efficiency of Gi or Wi alone in consideration that Wnt signaling pathway has been shown to have biphasic effect on cardiac development in zebrafish, mouse embryos, and mouse embryonic stem cells with early Wnt signaling enhancing and later Wnt signaling repressing heart development $[28,29]$, suggesting that the cardiogenic effect of Wnt signaling pathway is highly timing sensitive and neither Gi nor Wi alone is not enough for highly efficient cardiomyocyte differentiation, and then high-efficiency SANCLs enrichment via combined modulation of MP, FGF, and RA signaling pathways based on the system of Gi or Wi alone.

\section{Conclusion}

In summary, we used a developmental biology-guided approach to establish a transgene-independent highly

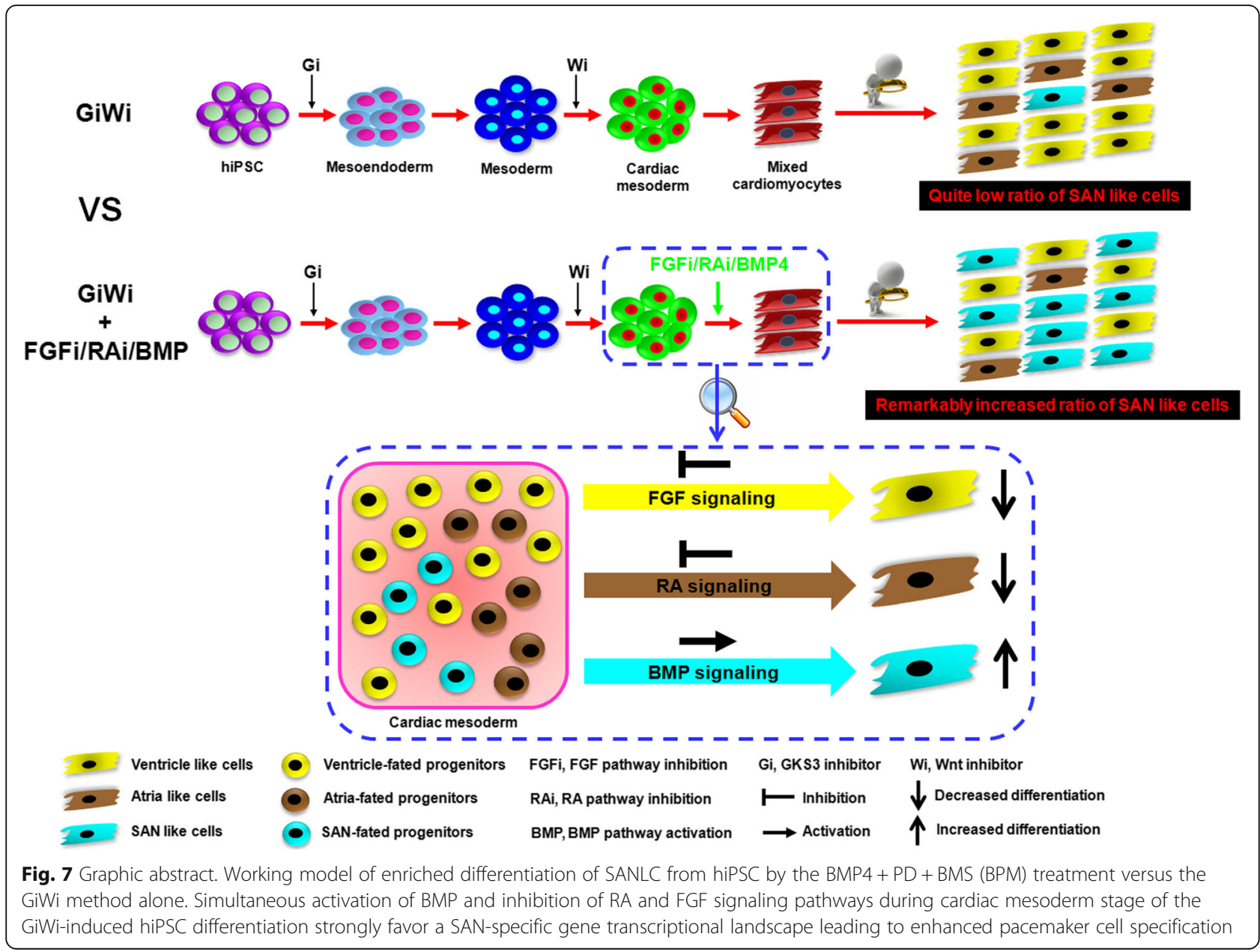


efficient differentiation protocol for generating SAN-like cardiomyocytes from hPSC. We found that activation of BMP signaling and simultaneous inhibitions of both RA and FGF pathways during cardiac mesoderm stage of hiPSC differentiation lead to a SAN-specific gene expression landscape favoring pacemaker cell specification (Fig. 7). This provides a rich source of SAN cardiomyocytes to further study its biology and the potential applications in the treatment of arrhythmia-related disease.

\section{Supplementary information}

Supplementary information accompanies this paper at https://doi.org/10. 1186/s13287-020-01794-5.

\section{Additional file 1: Suppl-Fig. 1. Validation of the enriched} differentiation of SANLC by the BMP4/PD/BMS (BPM). Representative IF analysis at day 21 showed that both $\mathrm{CTNT}^{+} / \mathrm{NKX}^{2} .5^{-}$and $\mathrm{CTNT}^{+} / \mathrm{SHOX2}^{+}$ populations representing SANLC accounted for around $50 \%$ and $40 \%$ of the total cells indicated by DAPI respectively, which is consistent with the corresponding flow cytometry analysis. Scale bars, $100 \mu \mathrm{m}$ (400x).

Additional file 2: Suppl-Fig. 2. Evaluation of the ventricular and atrial working cardiomyocytes in the BMP4/PD/BMS (BPM)-induced SANLC population. (A, B) Compared to the GiWi control, the expressions of specific atrial markers (MYL7 and COUPTFII) (A) and ventricle markers (MYL2 and NKX2.5) (B) were remarkably downregulated in BPM enriched SANLC at day 16 indicated by qPCR analysis (t test, ${ }^{*} p<0.05$ versus GiWi control, $n=3$ ). (C) Representative IF analysis further showed that quite low fractions of COUPTFII ${ }^{+}$and $\mathrm{MLC} \mathrm{V}^{+}$(corresponding MYL2 gene) population was observed in total cells indicated by DAPI. Scale bars, $100 \mu \mathrm{m}(400 \times)$. Expression values of all PCR analyses were normalized to the housekeeping gene GAPDH. Data are presented as 'Mean \pm SD'.

Additional file 3: Suppl-Fig. 3. Evaluation of the atrioventricular node (AVN) cells in the BMP4/PD/BMS (BPM)-induced SANLC population. Compared to the GiWi control, the expressions of specific AVN cells specific markers (MSX2 and TBX2) were significantly decreased in BPM group at day 16 as shown by qPCR analysis (t test, ${ }^{*} p<0.05$ versus GiWi control, $n=3$ ). Expression values of the PCR analysis was normalized to the housekeeping gene GAPDH. Data are presented as 'Mean \pm SD'.

Additional file 4: Suppl-Fig. 4. Evaluation of the expression of ion channel coding genes in the BMP4/PD/BMS (BPM)-induced SANLC population. A series of more other SANLC markers were checked using QPCR analysis 16 days after cell culture. (A) ISL1 (specific SANLC transcription factor) and HCN4 (SANLC specific pacemaker channel) were dramatically increased. (B-D) The expression of potassium, calcium and sodium ion channels distinguishing three types of cardiomyocytes (SANLC, ventricle and atria) were determined. (B) BMP treatment resulted in significant upregulation of SANLC potassium channel genes (KCND2, KCNK2 and KCNN4) in contrast with obvious decrease of ventricle (KCNQ1) and atria channels (KCNJ5). (C) Calcium ion channels related genes (CACNAIA and CACNB1) were increased by BPM while CACNA1C for ventricle was reduced. (D) BPM treatment remarkably increased the expression of SCN3B, SANLC specific sodium channel and decreased the level of SCN5, ventricle sodium channel. (E) Gap junction channel genes specific for SANLC (CX30.2), ventricle (CX43) and atria (CX40) were also tested. (t test, $* 0<0.05$ and ${ }^{*} p<0.01$ versus GiWi control, $n=3$ ). Expression values of all PCR analysis was normalized to the housekeeping gene GAPDH. Data are presented as 'Mean \pm SD'.

\section{Additional file 5: Suppl Video 1 \\ Additional file 6: Suppl Video 2 \\ Additional file 7: Suppl Video 3 \\ Additional file 8: Suppl Video 4}

\section{Abbreviations}

PSC: Pluripotent stem cell; hiPSC: Human induced pluripotent stem cell; SA: Sinoatrial; SANLC: Sinoatrial node-like cell; SSS: Sick sinus syndrome; AVN: Atrioventricular node; BMP: Bone morphogenetic protein;
FGF: Fibroblast growth factor; RA: Retinoic acid; IF: Immunofluorescence; AP: Action potential; GiWi: GSK3 inhibitor and Wnt inhibitor

\section{Acknowledgements \\ N/A}

\section{Authors' contributions}

Guarantor of integrity of entire study: Rui Zhou and Bin Liao. Study concepts: Feng Liu, Rui Zhou, and Bin Liao. Study design: Linli Wang, Rui Zhou, and Bin Liao. Literature research: Feng Liu, Xiaojie Hou, and Yibing Fang.

Experimental studies: Feng Liu, Yibing Fang, Xiaojie Hou, Ying Yan, Haiying Xiao, Dongchuan Zuo, and Jing Wen. Data acquisition: Feng Liu and Yibing Fang. Data analysis/interpretation: Xiaojie Hou and Ying Yan. Statistical analysis: Ying Yan and Yibing Fang. Manuscript preparation: Feng Liu, Xiaojie Hou, and Rui Zhou. Manuscript editing: Xitong Dang, Zhichao Zhou, and Linli Wang. Manuscript revision/review: Xitong Dang, Zhichao Zhou, and Rui Zhou. Manuscript final version approval: Rui Zhou and Bin Liao. The authors read and approved the final manuscript.

\section{Funding}

This work was supported by grants from the collaborative innovation center for prevention and treatment of cardiovascular disease of Sichuan province [xtcx-2019-02 to B. L., xtcx-2016-18 to R. Z.].

Availability of data and materials

The datasets used and/or analyzed during the current study are available from the corresponding author on reasonable request.

Ethics approval and consent to participate

Not applicable

Consent for publication

Not applicable

\section{Competing interests}

The authors declare that they have no competing interests.

\section{Author details}

'Department of Cardiac Macrovascular Surgery, Affiliated Hospital of Southwest Medical University, 3-319 Zhongshan Road, Luzhou 646000, Sichuan, China. ${ }^{2}$ College of Integrated Traditional Chinese and Western Medicine, Southwest Medical University, Luzhou 646000, Sichuan, China. ${ }^{3}$ The Key Laboratory of Medical Electrophysiology of Ministry of Education and Medical Electrophysiological Key Laboratory of Sichuan Province, Collaborative Innovation Center for Prevention and Treatment of Cardiovascular Disease of Sichuan Province, Institute of Cardiovascular Research, Southwest Medical University, 3-319 Zhongshan Road, Luzhou 646000, Sichuan, China. ${ }^{4}$ Guangzhou Biocare Institute of Cancer, Guangzhou 510663, Guangdong, China. ${ }^{5}$ Division of Cardiology, Department of Medicine, Karolinska University Hospital, Karolinska Institute, Stockholm, Sweden.

Received: 13 February 2020 Revised: 15 June 2020

Accepted: 29 June 2020 Published online: 16 July 2020

\section{References}

1. Li R. Gene- and cell-based bio-artificial pacemaker: what basic and translational lessons have we learned? Gene Ther. 2012;19:588-95.

2. Jung JJ, Husse B, Rimmbach C, Krebs S, Stieber J, Steinhoff G, et al. Programming and isolation of highly pure physiologically and pharmacologically functional sinus-nodal bodies from pluripotent stem cells. Stem Cell Reports. 2014;2:592-605.

3. Später D, Abramczuk MK, Buac K, Zangi L, Stachel MW, Clarke J, et al. A $\mathrm{HCN} 4+$ cardiomyogenic progenitor derived from the first heart field and human pluripotent stem cells. Nat Cell Biol. 2013;15:1098-106.

4. Lescroart F, Chabab S, Lin X, Rulands S, Paulissen C, Rodolosse A, et al. Early lineage restriction in temporally distinct populations of Mesp1 progenitors during mammalian heart development. Nat Cell Biol. 2014;16:829-40.

5. Später D, Hansson E, Zangi L, Chien K. How to make a cardiomyocyte. Development. 2014;141:4418-31.

6. Ma J, Guo L, Fiene SJ, Anson BD, Thomson JA, Kamp TJ, et al. High purity human-induced pluripotent stem cell-derived cardiomyocytes: 
electrophysiological properties of action potentials and ionic currents. Am J Physiol Heart Circ Physiol. 2011;301:H2006-17.

7. Blazeski A, Zhu R, Hunter DW, Weinberg SH, Boheler KR, Zambidis ET, Tung L. Electrophysiological and contractile function of cardiomyocytes derived from human embryonic stem cells. Prog Biophys Mol Biol. 2012;110:178-95.

8. Lee J, Protze S, Laksman Z, Backx P, Keller G. Human pluripotent stem cellderived atrial and ventricular cardiomyocytes develop from distinct mesoderm populations. Cell Stem Cell. 2017;21:179-94.

9. Zhang Q, Jiang J, Han P, Yuan Q, Zhang J, Zhang X, et al. Direct differentiation of atrial and ventricular myocytes from human embryonic stem cells by alternating retinoid signals. Cell Res. 2011;21:579-87.

10. Mommersteeg MT, Hoogaars WM, Prall OW, de Gier-de Vries C, Wiese C, Clout $\mathrm{DE}$, et al. Molecular pathway for the localized formation of the sinoatrial node. Circ Res. 2007;100:354-62.

11. Mommersteeg MT, Domínguez JN, Wiese C, Norden J, de Gier-de Vries C, Burch JB, et al. The sinus venosus progenitors separate and diversify from the first and second heart fields early in development. Cardiovasc Res. 2010; 87:92-101.

12. Wiese C, Grieskamp T, Airik R, Mommersteeg MT, Gardiwal A, de Gier-de Vries $C$, et al. Formation of the sinus node head and differentiation of sinus node myocardium are independently regulated by Tbx18 and Tbx3. Circ Res. 2009;104:388-97.

13. Targoff KL, Colombo S, George V, Schell T, Kim SH, Solnica-Krezel L, Yelon D. Nkx genes are essential for maintenance of ventricular identity. Development. 2013;140:4203-13.

14. George V, Colombo S, Targoff K. An early requirement for $n k \times 2.5$ ensures the first and second heart field ventricular identity and cardiac function into adulthood. Dev Biol. 2015;400:10-22.

15. Targoff $K$, Schell T, Yelon D. Nkx genes regulate heart tube extension and exert differential effects on ventricular and atrial cell number. Dev Biol. 2008; 322:314-21.

16. Dorn T, Goedel A, Lam J, et al. Direct nkx2-5 transcriptional repression of is 11 controls cardiomyocyte subtype identity. Stem Cells. 2015;33:1113-29.

17. Klaus A, Saga Y, Taketo M, Tzahor E, Birchmeier W. Distinct roles of Wnt/ beta-catenin and Bmp signaling during early cardiogenesis. Proc Natl Acad Sci U S A. 2007:104:18531-6.

18. Witty AD, Mihic A, Tam RY, Fisher SA, Mikryukov A, Shoichet MS, et al. Generation of the epicardial lineage from human pluripotent stem cells. Nat Biotechnol. 2014;32:1026-35.

19. Lian X, Zhang J, Azarin SM, Zhu K, Hazeltine LB, Bao X, et al. Directed cardiomyocyte differentiation from human pluripotent stem cells by modulating Wnt/ $\beta$-catenin signaling under fully defined conditions. Nat Protoc. 2013;8:162-75.

20. El-Battrawy I, Zhao Z, Lan H, Cyganek L, Tombers C, Li X, et al. Electrical dysfunctions in human-induced pluripotent stem cell-derived cardiomyocytes from a patient with an arrhythmogenic right ventricular cardiomyopathy. Europace. 2018;20:46-56.

21. Gorabi AM, Hajighasemi S, Tafti HA, Atashi A, Soleimani M, Aghdami N, et al. TBX18 transcription factor overexpression in human-induced pluripotent stem cells increases their differentiation into pacemaker-like cells. J Cell Physiol. 2019;234:1534-46.

22. Kapoor N, Liang W, Marbán E, Cho H. Direct conversion of quiescent cardiomyocytes to pacemaker cells by expression of Tbx18. Nat Biotechnol. 2013;31:54-62.

23. Yang $M$, Zhang GG, Wang $T$, Wang $X$, Tang $Y H$, Huang $H$, et al. TBX18 gene induces adipose-derived stem cells to differentiate into pacemaker-like cells in the myocardial microenvironment. Int J Mol Med. 2016;38:1403-10

24. Protze SI, Liu J, Nussinovitch U, Ohana L, Backx PH, Gepstein L, et al. Sinoatrial node cardiomyocytes derived from human pluripotent cells function as a biological pacemaker. Nat Biotechnol. 2017;35:56-68

25. Yang L, Soonpaa MH, Adler ED, Roepke TK, Kattman SJ, Kennedy M, et al. Human cardiovascular progenitor cells develop from a KDR+ embryonicstem-cell-derived population. Nature. 2008;453:524-8.

26. Pradhan A, Zeng XI, Sidhwani P, Marques SR, George V, Targoff KL, et al. FGF signaling enforces cardiac chamber identity in the developing ventricle. Development. 2017;144:1328-38.

27. Birket MJ, Ribeiro MC, Verkerk AO, Ward D, Leitoguinho AR, den Hartogh SC, et al. Expansion and patterning of cardiovascular progenitors derived from human pluripotent stem cells. Nat Biotechnol. 2015;33:970-9.
28. Ueno S, Weidinger G, Osugi T, Kohn AD, Golob JL, Pabon L, et al. Biphasic role for Wnt/beta-catenin signaling in cardiac specification in zebrafish and embryonic stem cells. Proc Natl Acad Sci. 2007;104:9685-90.

29. Naito AT, Shiojima I, Akazawa H, Hidaka K, Morisaki T, Kikuchi A, et al. Developmental stage-specific biphasic roles of Wnt/beta-catenin signaling in cardiomyogenesis and hematopoiesis. Proc Natl Acad Sci. 2006;103: 19812-7.

\section{Publisher's Note}

Springer Nature remains neutral with regard to jurisdictional claims in published maps and institutional affiliations.
Ready to submit your research? Choose BMC and benefit from:

- fast, convenient online submission

- thorough peer review by experienced researchers in your field

- rapid publication on acceptance

- support for research data, including large and complex data types

- gold Open Access which fosters wider collaboration and increased citations

- maximum visibility for your research: over $100 \mathrm{M}$ website views per year

At BMC, research is always in progress.

Learn more biomedcentral.com/submissions 